\title{
A cross-sectional study: serum CCL3/MIP-I $\alpha$ levels may reflect lumbar intervertebral disk degeneration in Han Chinese people
}

This article was published in the following Dove Press journal: Journal of Pain Research

\author{
Yi-Li Zhang ${ }^{1,2, *}$ \\ Bei $\mathrm{Li}^{1,2, *}$ \\ Zeng-Huan Zhou' \\ 'School of Public Health, Southern \\ Medical University, Guangzhou, \\ Guangdong Province, People's \\ Republic of China; ${ }^{2}$ School of Health \\ Services Management, Southern \\ Medical University, Guangzhou, \\ Guangdong Province, People's \\ Republic of China
}

*These authors contributed equally to this work

Correspondence: Zeng-Huan Zhou School of Public Health, Southern Medical University, ShaTai South Road No.1023, Bai Yun District, Guangzhou, Guangdong Province 510515, People's Republic of China

Email zzhsmu@yeah.net
Background: The macrophage inflammatory protein-1 $\alpha$ (MIP-1 $\alpha)$, also named chemokine cytokine ligand 3 (CCL3), has been detected in nucleus pulposus and increased following cytokine stimulation.

Objective: The current study was performed to explore the relationship between serum CCL3/ MIP-1 $\alpha$ levels with lumbar intervertebral disk degeneration (IDD).

Patients and methods: A total of 132 disk degeneration patients confirmed by magnetic resonance imaging and 126 healthy controls were enrolled in the current study. Radiological evaluation of the IDD was conducted using a 3.0-T magnetic resonance imaging scanner for entire lumbar vertebra region. Degeneration of intervertebral disk was assessed by Schneiderman criteria. Serum CCL3/MIP-1 $\alpha$ levels were investigated using a sandwich enzyme-linked immunosorbent assay. The Visual Analog Scale scores and Oswestry Disability Index index were recorded for clinical severity.

Results: Elevated concentrations of CCL3 in serum were found in IDD patients compared with asymptomatic volunteers. The case group included 49 IDD patients with grade 1, 42 with grade 2 , and 41 with grade 3 . Grade 3 and 2 had significantly higher CCL3 concentrations in serum compared with those with grade 1 . The serum CCL3 levels were positively related to the degree of disk degeneration. In addition, the serum CCL3 levels also demonstrated a significant correlation with the clinical severity determined by Visual Analog Scale scores and Oswestry Disability Index index.

Conclusion: Serum CCL3 may serve as a biomarker of IDD.

Keywords: chemokine cytokine ligand 3, intervertebral disk degeneration, cross-sectional study

\section{Introduction}

Low back pain (LBP) has an extremely high incidence. It is estimated that nearly $84 \%$ of the worldwide population experience LBP during their lifetime, with $10 \%$ being chronically disabled. ${ }^{1}$ LBP severely influences the work and impairs the quality of life, resulting in significant social and economical burdens. ${ }^{2}$ The etiological factors of LBP are various, among which, lumbar intervertebral disc degeneration (IDD) is a leading cause of lumbar spine-originated LBP. ${ }^{3}$

Disk degeneration is characterized by a loss of cellularity and degradation of the extracellular matrix resulting in morphologic changes and alterations in biomechanical properties. ${ }^{4}$ Several imaging techniques have been utilized to detect IDD. Radiography and conventional computed tomography and magnetic resonance imaging (MRI) are the three common imaging methods for evaluation of disk morphology and reactive bony changes. ${ }^{5-7}$ However, the biochemical events during early IDD, including 
the breakdown of large aggregating proteoglycan gels and extracellular matrix degradation, occur before the morphologic changes and alterations in biomechanical properties. In addition, there are currently no definite ways to instantly monitor the condition of disk degeneration. ${ }^{8}$ Therefore, it is necessary to seek more effective, reliable, and rapid methods to monitor early disk degeneration.

Biomarker is objectively measured and evaluated as an indicator of normal biologic processes, pathogenic processes, or pharmacologic responses to a therapeutic intervention. ${ }^{9}$ The use of biomarkers in various fields in the past decades has been widely proposed. When referring to disk degeneration, analysis of biomarkers could serve as a clinically relevant method before signs of degeneration are visible by radiology assessment. ${ }^{10}$

Chemokines consist of a large family of small proteins and generally have a low molecular weight ranging from 7 to $15 \mathrm{kDa}$ and were first described as chemoattractant cytokines synthesized at sites of inflammation and are the regulatory proteins for leukocyte recruitment and trafficking. ${ }^{11} \mathrm{Chemo-}$ kines are subdivided into four subfamilies, C-, CC-, CXC-, and $\mathrm{CX} 3 \mathrm{C}$-chemokine, based on the number and spacing of the first two cysteines in a conserved cysteine structural motif. ${ }^{12}$ Chemokines have been reported to participate various inflammation and autoimmune disease including osteoarthritis, rheumatoid arthritis, etc. ${ }^{13}$ Recently, chemokines have also been detected in tissue and serum in disk degeneration disease, ${ }^{14,15}$ and may exert a pivotal role in disk degeneration. Macrophage inflammatory protein- $1 \alpha$ (MIP-1 $\alpha$, also known as CCL3) is a member of the CC chemokine family. CCL3 has been extensively studied since its initial identification as a macrophage-derived inflammatory mediator. ${ }^{16}$ In one previous study, CCL3 has been detected in herniated intervertebral discs, ${ }^{17}$ and the expression and activity was related to fibroblasts, endothelial cells, and infiltrating macrophages in the granulation tissues. ${ }^{17}$ In another bioinformatic analysis of the microarray data on the gene expression profiles of degenerative intervertebral disc cells after exposure to TNF- $\alpha$, CCL3 was shown to play a crucial role in the development of the disc degeneration. ${ }^{18}$

All these studies above suggest that CCL3 may play an important role in disc degeneration. To our knowledge, there were no studies available illustrating the relationship between serum CCL3 concentrations and disease severity in IDD. Therefore, we intended to investigate the serum expressions of CCL3 in IDD patients and their association with clinical severity, to determine whether CCL 3 could be used as a rapid and novel biomarker for diagnosis of disc degeneration.

\section{Patients and methods}

\section{Study patients}

From July 2016 to August 2017, a total of 132 patients diagnosed with lumbar IDD were enrolled in the current study. This study was approved by the ethics committee of Southern Medical University. The inclusion criteria of patients were the presence of significant back pain, which was defined as a pain in the low back for $>2$ weeks and severe enough to require physician consultation or treatment. ${ }^{19}$ Moreover, inclusion criteria of asymptomatic volunteers were done based on a report by Pfirrmann, ${ }^{20}$ who observed the process of normal aging in the lumbar disks, as follows: 1) no back pain within the last 3 years; 2) never absent from work because of LBP; 3 ) no history of consulting a physician because of LBP. One hundred and twenty six sex- and age-matched healthy volunteers receiving body check were recruited as controls. All the patients and healthy control individuals signed the informed consent form approved by the university ethics committee. The study design was in accordance with the Declaration of Helsinki guidelines.

\section{Laboratory investigation}

Peripheral venous blood was obtained from the antecubital vein following a 12 hours fasting period. Blood was collected using EDTA as an anticoagulant and centrifuged for 15 minutes at $1,000 \mathrm{~g}$ within 30 minutes of collection. Serum samples were aliquoted and stored at $-80^{\circ}$ until use. CCL3 concentrations in serum were examined using the human CCL3 Quantikine ELISA Kit (R\&D Systems, Minneapolis, MN, USA) according to the manufacturer's instructions. Briefly, standards of recombinant human CCL3 and serum samples were added to 96-well microtiter plates precoated with mouse monoclonal antibody against CCL3 and incubated for 2 hours at room temperature. The wells were then washed four times with washing buffer and incubated for 2 hours at room temperature with a horseradish peroxidaseconjugated goat polyclonal antibody against CCL3. After four washes, substrate solution was added to each well, and the plate was incubated for 30 minutes at room temperature in the dark. Finally, the reaction was stopped with the stop solution and absorbance was measured at $450 \mathrm{~nm}$ using an automated microplate reader. Recombinant human CCL3 was used to generate a linear standard calibration curve. All examinations were repeated at least three times.

\section{Radiological evaluation}

All participants underwent whole spine T2-weighted sagittal MRI without fat suppression. The MRI protocol was 
as follows: $5 \mathrm{~mm}$ slice thickness, $1 \mathrm{~mm}$ slice gap, FOV of $280 \times 240 \mathrm{~mm}^{2}$, and a matrix of $448 \times 336$. The degree of discs degeneration of the lumbar spine (L1-S1) was assessed according to the Schneiderman criteria ${ }^{21}$ for disc degeneration. This grading system scores from level 0 to 3: 0 level means no degeneration denoted by high signal intensity of the disc; 1 level represent mild degeneration with slight decrease in signal intensity of the nucleus pulposus; 2 levels indicate moderate degeneration with generalized hypointensity of the nucleus pulposus (black disc) with normal disc height; and 3 levels suggest severe degeneration (black disc) with disc height narrowing. Based on this classification scheme, each of the five lumbar discs was assigned a grade (potential range: $0-3$ ). The results of MRI evaluation were read by two radiology experts. The reliability of the MRI evaluations was assessed using agreement percentage and Kappa statistics. The agreement was rated as follows: Kappa value = $0-0.2$ indicated slight agreement, $0.21-0.4$ fair agreement, $0.41-0.60$ moderate agreement, $0.61-0.8$ substantial agreement, and 0.81 upward excellent agreement.

\section{Evaluation of symptomatic severity}

The symptomatic severity was evaluated by Visual Analog Scale (VAS) score to assess degree of pain and Oswestry Disability Index (ODI) index to evaluate functional disability. For VAS score, all the patients were asked to rate their pain intensity on a $10 \mathrm{~cm}$ VAS with end points "no pain" and "worst possible pain." The Oswestry Low Back Pain Disability Index is a 10-item self-assessing questionnaire, and each item contains 6 levels of answers that can be scored from 0 to 5. These items are: pain, personal care, lifting and moving objects, walking, sitting, standing, sleep disorders caused by the LBP, sex life, social life, and traveling. A total score is calculated, and percentage of disability (score obtained divided by 50 and multiplied by 100) ranges from 0 (no disability) to 100 (complete disability). The interpretation of this scale is based on the scores: from 0 to 20 : minimal disability; from 20 to 40: moderate disability; from 40 to 60: severe disability; from $60 \%$ to $80 \%$ crippling LBP; and beyond 80 : the person is confined to bed. ${ }^{22}$

\section{Statistical analysis}

Statistical calculations and analysis were carried out using GraphPad Prism 6.0 (GraphPad Software, La Jolla, CA, USA). The basic data were expressed as mean $\pm \mathrm{SD}$. The data of CCL 3 levels were presented as mean \pm standard error of mean. We determined the differences in CCL3 levels between the control and disease groups using Student's $t$-test or Mann-Whitney test. The Kolmogorov-Smirnov test was used to examine the normality of the distribution. The Spearman correlation test (nonparametric) was employed to examine the relationship between serum CCL3 concentrations and MRI grade of disc degeneration as well as VAS score and ODI index. $P$-values less than 0.05 were regarded as significant.

\section{Results}

\section{Baseline characteristics}

All data were obtained from a total of 132 patients including 67 men and 65 women (age ranging from 20 to 57 years) and 126 healthy controls including 63 males and 63 females (age ranging from 21 to 60 years). As shown in Table 1, there were no significant differences in baseline characteristics between IDD patients and healthy controls $(P>0.05)$ including age, sex distribution, height, and body mass index (Table 1). Elevated levels of CCL3 in serum were found in IDD patients compared with healthy controls $(277.3 \pm 4.3 \mathrm{pg} / \mathrm{mL}$ vs $150.2 \pm 2.4$ $\mathrm{pg} / \mathrm{mL}, P<0.001$ ) (Figure 1).

\section{Serum CCL3 levels in IDD patients with MRI severity defined by Schneiderman criteria}

The $\kappa$ value for radiographic assessment was 0.77 following evaluation. The CCL3 concentrations in serum of IDD patients with different Schneiderman MRI grades are

Table I Baseline clinical characteristics

\begin{tabular}{|c|c|c|c|}
\hline Items & IDD patients $(n=132)$ & Healthy controls $(n=126)$ & P-value \\
\hline Age (years) & $40.3 \pm 1.1$ & $41.2 \pm 0.6$ & 0.278 \\
\hline $\operatorname{Sex}(F / M)$ & $65 / 67$ & $63 / 63$ & 0.903 \\
\hline BMI $\left(\mathrm{kg} / \mathrm{m}^{2}\right)$ & $22.1 \pm 0.2$ & $22.3 \pm 0.3$ & 0.461 \\
\hline VAS score & $3.6 \pm 0.1$ & & \\
\hline ODI index & $24.1 \pm 0.7$ & & \\
\hline Schneiderman grading $(1 / 2 / 3)$ & $49 / 42 / 41$ & & \\
\hline Serum CCL3 levels (pg/mL) & $277.3 \pm 4.3$ & $150.2 \pm 2.4$ & $<0.001$ \\
\hline
\end{tabular}

Notes: Basic values and serum CCL3 levels are expressed as the mean value \pm SEM.

Abbreviations: IDD, intervertebral disk degeneration; BMI, body mass index; VAS, Visual Analog Scale; ODI, Oswestry Disability Index; SEM, standard error of the mean. 
depicted in Figure 2. With regard to the MRI severity of IDD, the patients were categorized into 3 groups according to the Schneiderman classification. The case group included 49 with grade 1, 42 with grade 2 , and 41 with grade 3 . IDD patients with grade 3 and grade 2 showed significantly elevated levels of CCL3 in serum compared with those with grade 1 (grade 3 vs grade $1: 300.6 \pm 6.6 \mathrm{pg} / \mathrm{mL}$ vs $250.7 \pm 6.2 \mathrm{pg} / \mathrm{mL}, P<0.001$; grade 2 vs grade $1: 285.5 \pm 7.4 \mathrm{pg} / \mathrm{mL}$ vs $250.7 \pm 6.2 \mathrm{pg} / \mathrm{mL}$, $P<0.001)$. The difference of CCL3 levels between Schneiderman 2 and 3 patients did not reach significance (grade 3 vs grade $2: 300.6 \pm 6.6 \mathrm{pg} / \mathrm{mL}$ vs $285.5 \pm 7.4 \mathrm{pg} / \mathrm{mL}, P=0.385$ ) (Figure 2A). The serum CCL3 levels were positively associated with Schneiderman grading system $(r=0.425, P<0.001)$ by Spearman's correlation analysis (Figure 2B).

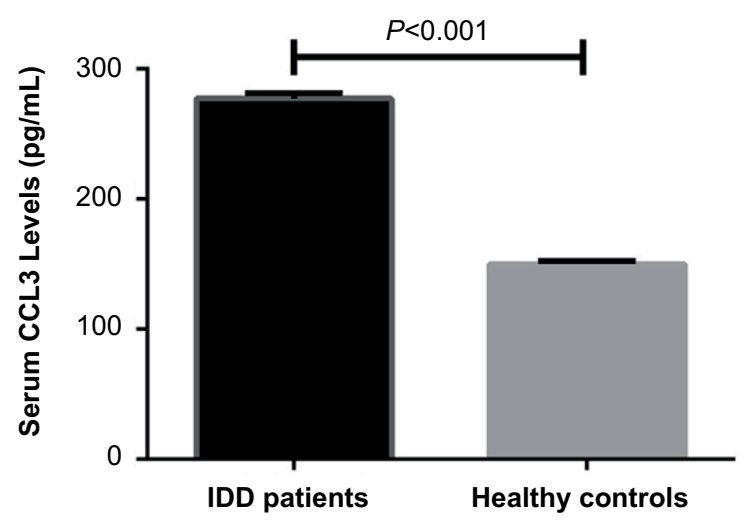

Figure I Serum CCL3 levels between IDD patients and healthy controls (mean \pm SEM).

Abbreviations: IDD, intervertebral disk degeneration; SEM, standard error of the mean.

\section{Serum CCL3 levels in IDD patients with clinical severity defined by VAS and ODI index}

Based on intensity of pain on a VAS $(0-10 \mathrm{~cm})$, the patients were divided into two groups, the high- and low-pain group with VAS $\geq 3$ and VAS $<3$, respectively. There were 76 patients in high-pain group and 56 patients in low-pain group. Serum CCL3 levels were significantly increased in high-pain group compared with low-pain group. (290.1 \pm 5.2 $\mathrm{pg} / \mathrm{mL}$ vs $259.9 \pm 6.6 \mathrm{pg} / \mathrm{mL}, P=0.013$ ) (Figure $3 \mathrm{~A}$ ). In addition, serum CCL3 concentrations were positively related to VAS scores $(r=0.395, P<0.001)$ (Figure 3B). We further explored the relationship between serum CCL3 levels and ODI index to illustrate whether higher levels of CCL3 would affect the functional ability in IDD patients. We found that serum CCL3 concentrations were also related to ODI index $(r=0.358, P<0.001)$ (Figure 3C).

\section{Discussion}

The current study investigated the relationship between serum CCL3 levels and degree of lumbar IDD. We demonstrated for the first time that increased serum CCL3 levels were observed in IDD patients compared with controls. In addition, CCL3 concentration in serum was independently and positively correlated with radiographic progression and clinical severity. These findings implicate that serum CCL3 may serve as a potential serum biomarker for reflecting lumbar IDD.

The increased number of people with IDD in recent years impelled the need for early diagnosis. Authors looking to
A

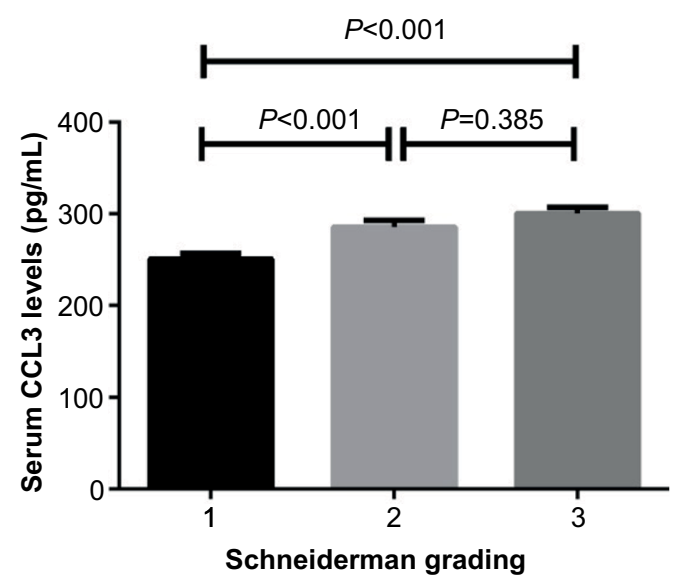

B

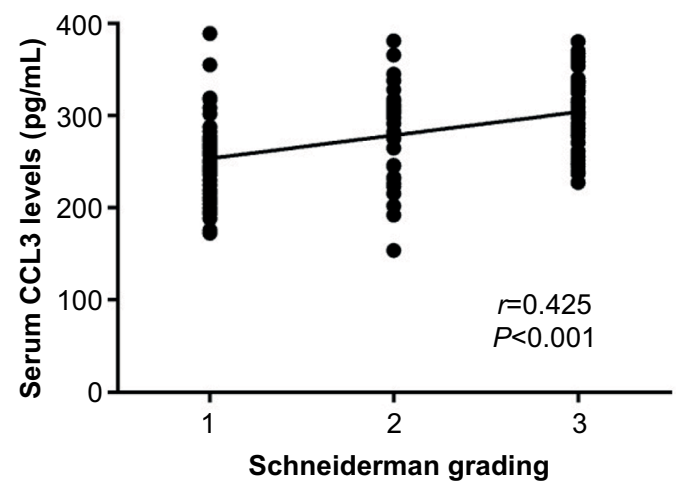

Figure 2 Correlation of serum CCL3 levels with radiographic severity in IDD patients

Notes: (A) Comparison of serum CCL3 levels among different Schneiderman MRI grades (mean \pm SEM). (B) Correlation of serum CCL3 levels with Schneiderman Grading. Abbreviations: IDD, intervertebral disk degeneration; MRI, magnetic resonance imaging; SEM, standard error of the mean. 
A

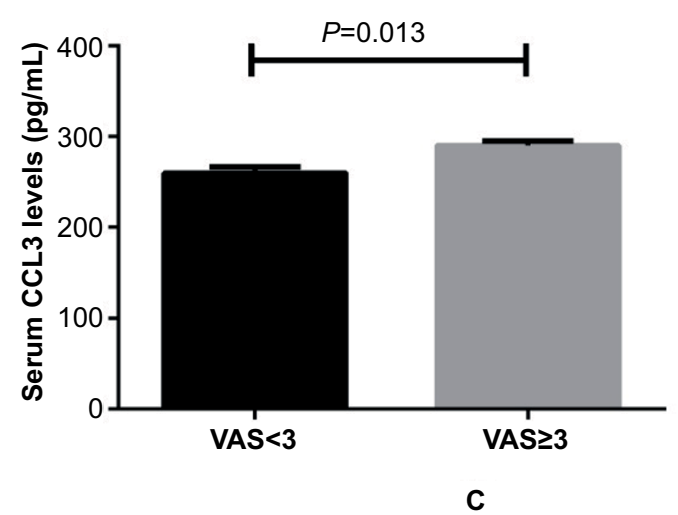

B

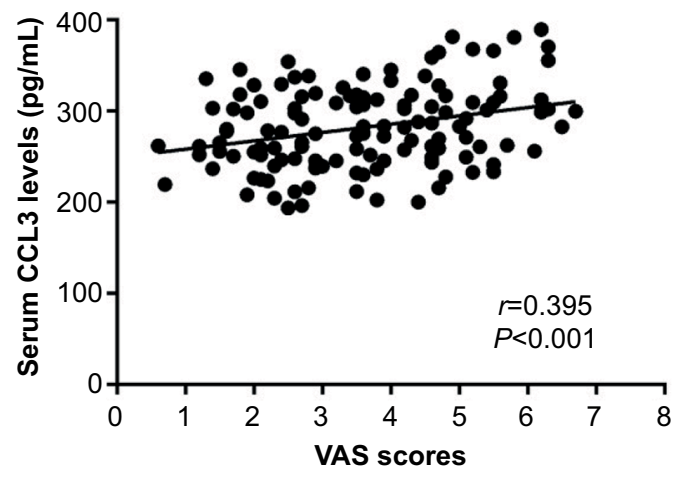

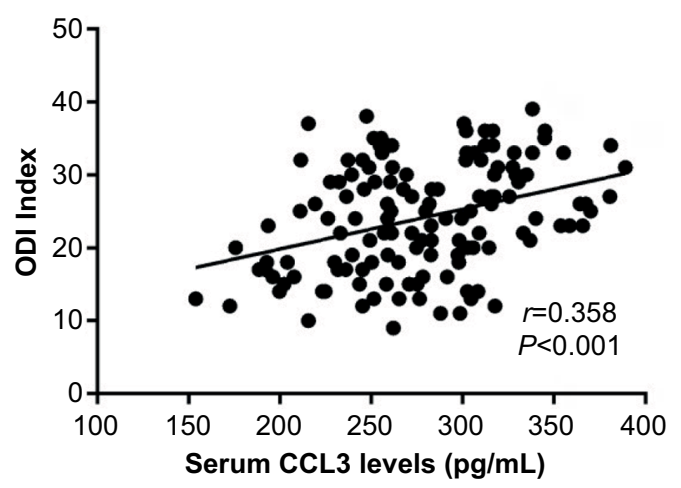

Figure 3 Relationship between serum CCL3 levels and VAS as well as ODI index in IDD patients

Notes: (A) Comparison of serum CCL3 levels between high-pain and low-pain patients (mean \pm SEM). (B) Correlation of serum CCL3 levels with VAS scores. (C) Correlation of serum CCL3 levels with ODI index.

Abbreviations: IDD, intervertebral disk degeneration; VAS, Visual Analog Scale; ODI, Oswestry Disability Index; SEM, standard error of the mean.

embrace a consensus of opinion related to the pathogenesis of IDD in their works have been devoting increasingly more attention to the importance of an inflammatory component. ${ }^{23}$ The expression of inflammatory mediators in the degenerated/herniated disks as well as their involvement in the process of disk degeneration (ie, in the induction of catabolic processes) has been investigated by both clinical studies as well as by laboratory investigation during the past decade. ${ }^{24,25}$ Identification of the relevance of these mediators in the development of discogenic back pain has led to an improved understanding of the molecular mechanisms of back pain, thus creating new possibilities of more targeted therapeutic intervention. ${ }^{26}$

The inflammatory chemokine CCL3 has been investigated in several studies addressing arthritic diseases. Plasma CCL3 could be a potential serum biomarker for knee osteoarthritis with the ability to detect pre-X-ray-defined changes and stage the severity of damage in knee..$^{27}$ In addition, expression and secretion of CCL3 by synovial fluid neutrophils may be indicative of local and systemic inflammation in rheumatoid arthritis. ${ }^{28}$ CCL 3 could be produced by different type of cells, and its production can be upregulated by the proinflammatory cytokines IL-1 and TNF- $\alpha .{ }^{29,30}$ More recently, CCL3 has been recognized as a chemotactic mediator produced by intervertebral disc cells. ${ }^{29,30}$

Pain is the main complain in IDD patients. In previous studies, elevated CCL3 levels have been detected in patients with neck/shoulder pain, migraine, and prostatitis pelvic pain, and these levels significantly correlated with pain intensity. ${ }^{31-33}$ In addition, in nonspecific patients with LBP, improvements in VAS and ODI scores were closely accompanied by the obvious decline of CCL3 following spinal manipulation. ${ }^{34}$ All these works implicate that systematic CCL3 levels may participate the pain production in various disease. In our study, we observed that increased CCL3 levels were related to pain determined by VAS scores and functional disability by ODI index, suggesting serum that CCL3 expression plays a major role in the pain formation of disk degeneration through potential mechanisms.

MR imaging can reflect both the macromolecular concentrations and the structural integrity in the intervertebral disk and has been considered as a noninvasive method of characterizing IDD in humans and animal models in vivo. ${ }^{35,36}$ However, previous studies have also noted that a relationship 
between disc degeneration and LBP is a coincidental finding and that the predictive capacity of MRI in terms of LBP is questionable. ${ }^{37,38} \mathrm{We}$ found increased serum CCL3 levels were positively related to MR imaging by Schneiderman grading, implicating CCL3 as a diagnosis biomarker in combination with the radiographic changes.

\section{Limitations}

Several potential limitations should be taken into account in our study. First, our study was based on a small sample size of Han Chinese people. A further study conducted on a random sample of a larger population will be needed to substantiate our results. Second, only serum CCL3 concentration has been measured in the serum. Additional immunohistochemical investigations of CCL3 expression in lumbar disk annulus fibrosus or nucleus pulposus may provide complete information on the pathogenic role of CCL3 in IDD. Third, we did not investigate the role of other soluble chemokines.

\section{Conclusion}

Collectively, in spite of the abovementioned limitations, the study revealed a significant elevation in serum CCL3 levels of IDD subjects compared with healthy controls and illustrated a pronounced positive correlation of serum CCL3 levels with the extent of radiographic and symptomatic severity in IDD patients. This study is the first to show that serum CCL3 is related to the disease progression of IDD. Further investigations are needed to define the mechanisms underlying this correlation. Therapies targeting CCL3 deserve further intensive study.

\section{Acknowledgments}

Funding for this work was provided by The 12th Five-year Plan project of Philosophy and Social Science in Guangdong Province (GD12CGL13) and The Project of School of Health Services Management of Southern Medical University (WG2016018).

\section{Disclosure}

The authors report no conflicts of interest in this work.

\section{References}

1. Patrick N, Emanski E, Knaub MA. Acute and chronic low back pain. Med Clin North Am. 2016;100:169-181.

2. Hoy D, Brooks P, Blyth F, et al. The epidemiology of low back pain. Best Pract Res Clin Rheumatol. 2010;24:769-781.

3. Thiese MS, Hegmann KT, Wood EM, et al. Prevalence of low back pain by anatomic location and intensity in an occupational population. $B M C$ Musculoskelet Disord. 2014;15:283.
4. Kepler CK, Anderson DG, Tannoury C, et al. Intervertebral disk degeneration and emerging biologic treatments. J Am Acad Orthop Surg. 2011;19:543-553.

5. Luoma K, Riihimaki H, Luukkonen R, et al. Low back pain in relation to lumbar disc degeneration. Spine (Phila Pa 1976). 2000;25:487-492.

6. Zuo J, Saadat E, Romero A, et al. Assessment of intervertebral disc degeneration with magnetic resonance single-voxel spectroscopy. Magn Reson Med. 2009;62:1140-1146.

7. Modic MT, Ross JS. Lumbar degenerative disk disease. Radiology. 2007;245:43-61.

8. Brinjikji W, Luetmer PH, Comstock B, et al. Systematic literature review of imaging features of spinal degeneration in asymptomatic populations. Am J Neuroradiol. 2015;36:811-816.

9. Biomarkers Definitions Working Group. Biomarkers and surrogate endpoints: preferred definitions and conceptual framework. Clin Pharmacol Ther. 2001;69:89-95.

10. Poole AR. Biologic markers and disc degeneration. J Bone Joint Surg Am. 2006;88:72-75.

11. Cameron MJ, Kelvin DJ. Cytokines and chemokines--their receptors and their genes: an overview. Adv Exp Med Biol. 2003;520:8-32.

12. Kunkel SL, Lukacs N, Strieter RM. Chemokines and their role in human disease. Agents Actions Suppl. 1995;46:11-22.

13. Santamaria P. Cytokines and chemokines in autoimmune disease: an overview. Adv Exp Med Biol. 2003;520:1-7.

14. Grad S, Bow C, Karppinen J, et al. Systemic blood plasma CCL5 and CXCL6: potential biomarkers for human lumbar disc degeneration. Eur Cell Mater. 2016;31:1-10.

15. Schroeder M, Viezens L, Schaefer C, et al. Chemokine profile of disc degeneration with acute or chronic pain. J Neurosurg Spine. 2013;18: 496-503.

16. Menten P, Wuyts A, Van Damme J. Macrophage inflammatory protein-1. Cytokine Growth Factor Rev. 2002;13:455-481.

17. Kawaguchi S, Yamashita T, Katahira G, et al. Chemokine profile of herniated intervertebral discs infiltrated with monocytes and macrophages. Spine. 2002;27:1511-1516.

18. Phillips KL, Chiverton N, Michael AL, et al. The cytokine and chemokine expression profile of nucleus pulposus cells: implications for degeneration and regeneration of the intervertebral disc. Arthritis Res Ther. 2013;15:R213.

19. Cheung KM, Karppinen J, Chan D, et al. Prevalence and pattern of lumbar magnetic resonance imaging changes in a population study of one thousand forty-three individuals. Spine. 2009;34:934-940.

20. Pfirrmann CW, Metzdorf A, Elfering A, et al. Effect of aging and degeneration on disc volume and shape: a quantitative study in asymptomatic volunteers. J Orthop Res. 2006;24:1086-1094.

21. Schneiderman G, Flannigan B, Kingston S, et al. Magnetic resonance imaging in the diagnosis of disc degeneration: correlation with discography. Spine. 1987;12:276-281.

22. Fairbank JC, Couper J, Davies JB, et al. The Oswestry low back pain disability questionnaire. Physiotherapy. 1980;66:271-273.

23. Sun Z, Zhang M, Zhao XH, et al. Immune cascades in human intervertebral disc: the pros and cons. Int J Clin Exp Pathol. 2013;15:1009-1014.

24. Podichetty VK. The aging spine: the role of inflammatory mediators in intervertebral disc degeneration. Cell Mol Biol (Noisy-le-grand). 2007;53:4-18.

25. Risbud MV, Shapiro IM. Role of cytokines in intervertebral disc degeneration: pain and disc content. Nat Rev Rheumatol. 2014;10:44-56.

26. Shamji MF, Setton LA, Jarvis W, et al. Proinflammatory cytokine expression profile in degenerated and herniated human intervertebral disc tissues. Arthritis Rheum. 2010;62:1974-1982.

27. Zhao XY, Yang ZB, Zhang ZJ, et al. CCL3 serves as a potential plasma biomarker in knee degeneration (osteoarthritis). Osteoarthritis Cartilage. 2015;23:1405-1411.

28. Hatano Y, Kasama T, Iwabuchi H, et al. Macrophage inflammatory protein 1 alpha expression by synovial fluid neutrophils in rheumatoid arthritis. Ann Rheum Dis. 1999;58:297-302. 
29. Wang J, Tian Y, Phillips KL, et al. Tumor necrosis factor $\alpha$ - and interleukin-1 $\beta$-dependent induction of CCL3 expression by nucleus pulposus cells promotes macrophage migration through CCR1. Arthritis Rheum. 2013;65:832-842.

30. Liu C, Fei HD, Sun ZY, et al. Bioinformatic analysis of the microarray gene expression profile in degenerative intervertebral disc cells exposed to TNF- $\alpha$. Eur Rev Med Pharmacol Sci. 2015;19:3332-3339.

31. Matute Wilander A, Kåredal M, Axmon A, et al. Inflammatory biomarkers in serum in subjects with and without work related neck/shoulder complaints. BMC Musculoskelet Disord. 2014;15:103.

32. Domingues RB, Duarte H, Senne C, et al. Serum levels of adiponectin, CCL3/MIP-1 $\alpha$, and CCL5/RANTES discriminate migraine from tension-type headache patients. Arq Neuropsiquiatr. 2016;74: 626-631.

33. Quick ML, Mukherjee S, Rudick CN, et al. CCL2 and CCL3 are essential mediators of pelvic pain in experimental autoimmune prostatitis. $\mathrm{Am}$ J Physiol Regul Integr Comp Physiol. 2012;303:R580-R589.
34. Teodorczyk-Injeyan JA, McGregor M, Triano JJ, et al. Elevated production of nociceptive CC-chemokines and sE-selectin in patients with low back pain and the effects of spinal manipulation: a non-randomized clinical trial. Clin J Pain. Epub 2017 Apr 19.

35. Majumdar S, Link TM, Steinbach LS, et al. Diagnostic tools and imaging methods in intervertebral disk degeneration. Orthop Clin North Am. 2011;42:501-511.

36. Jeffery ND, Levine JM, Olby NJ, et al. Intervertebral disk degeneration in dogs: consequences, diagnosis, treatment, and future directions. JVet Intern Med. 2013;27:1318-1333.

37. Borenstein DG, O'Mara JW Jr, Boden SD, et al. The value of magnetic resonance imaging of the lumbar spine to predict low-back pain in asymptomatic subjects: a seven-year follow-up study. J Bone Joint Surg Am. 2001;83:1306-1311.

38. Jensen MC, Brant-Zawadzki MN, Obuchowski N, et al. Magnetic resonance imaging of the lumbar spine in people without back pain. N Engl J Med. 1994;331:69-73.

\section{Journal of Pain Research}

\section{Publish your work in this journal}

The Journal of Pain Research is an international, peer reviewed, open access, online journal that welcomes laboratory and clinical findings in the fields of pain research and the prevention and management of pain. Original research, reviews, symposium reports, hypothesis formation and commentaries are all considered for publication

\section{Dovepress}

The manuscript management system is completely online and includes a very quick and fair peer-review system, which is all easy to use. Visit http://www.dovepress.com/testimonials.php to read real quotes from published authors. 